\title{
Identification of Adomavirus Virion Proteins
}

5 Nicole L. Welch ${ }^{1}$, Michael J. Tisza ${ }^{1}$, Gabriel J. Starrett ${ }^{1}$, Anna K. Belford ${ }^{1}$, Diana V. Pastrana ${ }^{1}$,

6 Yuk-Ying S. Pang ${ }^{1}$, John T. Schiller ${ }^{1}$, Ping An², Paul G. Cantalupo ${ }^{2}$, James M. Pipas ${ }^{2}$, Samantha

$7 \mathrm{Koda}^{3}$, Kuttichantran Subramaniam ${ }^{3}$, Thomas B. Waltzek ${ }^{3}$, Chao Bian ${ }^{4}$, Qiong Shi ${ }^{4}$, Zhiqiang

$8 \quad$ Ruan ${ }^{4}$, Terry Fei Fan $\mathrm{Ng}^{5}$, and Christopher B. Buck ${ }^{1 *}$

$10{ }^{1}$ Lab of Cellular Oncology, NCI, NIH, Bethesda, MD, 20892 USA

112 Dept. of Biological Sciences, Univ. of Pittsburgh, Pittsburgh, PA 15260 USA

$12{ }^{3}$ Dept. of Infectious Disease and Pathology, Univ. of Florida, Gainesville, FL, 32611 USA

$13{ }^{4}$ Shenzhen Key Lab of Marine Genomics, Guangdong Provincial Key Lab of Molecular Breeding 14 in Marine Economic Animals, BGI Academy of Marine Sciences, BGI, Shenzhen, Guangdong 15 518083, China

$16{ }^{5}$ College of Veterinary Medicine, University of Georgia, Athens, GA 30602 USA

17

18 *Corresponding author: buckc@mail.nih.gov 


\section{Abstract}

Adenoviruses, papillomaviruses, and polyomaviruses are collectively known as small DNA tumor viruses. Although it has long been recognized that small DNA tumor virus oncoproteins and capsid proteins show a variety of structural and functional similarities, it is unclear whether these similarities reflect descent from a common ancestor, convergent evolution, horizontal gene transfer among virus lineages, or acquisition of genes from host cells. Here, we report the discovery of a dozen new members of an emerging virus family, the Adomaviridae, that unite a papillomavirus/polyomavirus-like replicase gene with an adenovirus-like virion maturational protease. Adomaviruses were initially discovered in a lethal disease outbreak among endangered Japanese eels. New adomavirus genomes were found in additional commercially important fish species, such as tilapia, as well as in reptiles. The search for adomavirus sequences also revealed an additional candidate virus family, which we refer to as xenomaviruses, in mollusk datasets. Analysis of native adomavirus virions and expression of recombinant proteins showed that the virion structural proteins of adomaviruses are homologous to those of both adenoviruses and another emerging animal virus family called adintoviruses. The results pave the way toward development of vaccines against adomaviruses and suggest a framework that ties small DNA tumor viruses into a shared evolutionary history.

\section{Author Summary}

In contrast to cellular organisms, viruses do not encode any universally conserved genes. Even within a given family of viruses, the amino acid sequences encoded by homologous genes can diverge to the point of unrecognizability. Although members of an emerging virus family, the Adomaviridae, encode replicative DNA helicase proteins that are recognizably similar to those of polyomaviruses and papillomaviruses, the functions of other adomavirus genes have been difficult to identify. Using a combination of laboratory and bioinformatic approaches, we identify the adomavirus virion structural proteins. The results link adomavirus virion protein operons to those of other midsize non-enveloped DNA viruses, including adenoviruses and adintoviruses. 
50 Polyomaviruses, papillomaviruses, and adenoviruses are historically defined as small DNA tumor viruses (Pipas 2019). Although members of a fourth animal-tropic non-enveloped DNA virus family, the Parvoviridae, are not known to cause tumors they share a number of biological features with traditional small DNA tumor viruses. Each of the four virus families encodes nonenveloped virion proteins with similar pentameric single- $\beta$-jellyroll core folds and members of each of the four families express functionally similar oncogenes that inactivate cellular tumor suppressor proteins (Figure 1)(de Souza, Iyer et al. 2010, Krupovic and Koonin 2017). An emerging group of animal viruses called adintoviruses appears to represent a candidate fifth family with similarities to small DNA tumor viruses https://www.biorxiv.org/content/10.1101/697771v3.
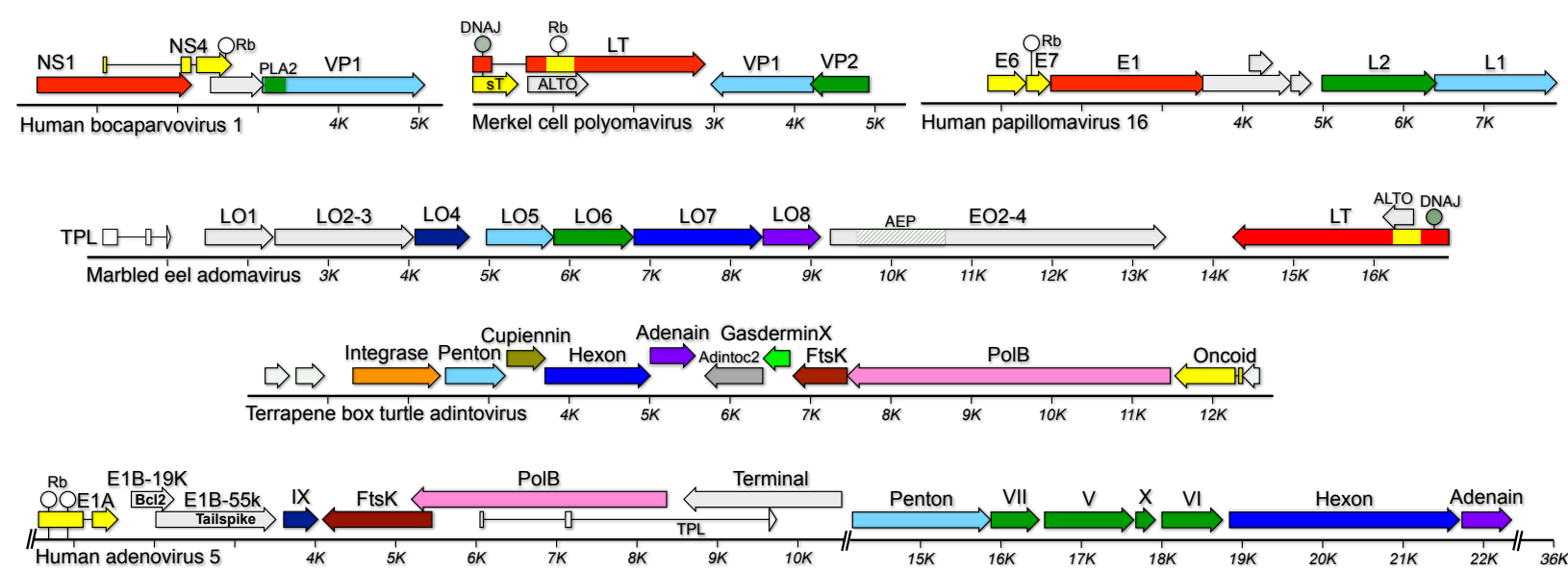

Figure 1: Maps of representative virus genomes. Genes are color-coded based on known or inferred functions. Polyomaviruses, papillomaviruses, and adomaviruses have circular double-stranded DNA genomes that were linearized for display. Parvoviruses have linear single-stranded DNA (ssDNA) genomes, while adintoviruses and adenoviruses have linear dsDNA genomes. Symbols and abbreviations: white lollipop, retinoblastoma/pocket protein $(\mathrm{Rb})$ interaction motif; gray lollipop, domain with predicted fold similar to DNAJ chaperone proteins; AEP, domain resembling archael-eukaryotic primase small catalytic subunit; PLA2, domain with predicted fold similar to phospholipase A2. See main text for other gene names.

Polyomaviruses, papillomaviruses, and parvoviruses are proposed to have descended from circular Rep-encoding single-stranded DNA (CRESS) virus ancestors (Koonin, Dolja et al. 2015). This model explains the phylogenetic relationships of the replicative superfamily 3 helicase ( $\mathrm{S} 3 \mathrm{H})$ and rolling circle "nickase" endonuclease domain of CRESS virus and small DNA tumor virus replicase genes (Kazlauskas, Varsani et al. 2019), but it does not account for possible similarities between the virion proteins and accessory genes of the "-oma" families and adenoviruses. Achieving a better understanding of the relationships between small DNA tumor virus families has the potential to guide comparative studies of these common human pathogens.

78 In 2011, a previously unknown circular dsDNA virus was discovered in a lethal disease outbreak 79 among Japanese eels (Anguilla japonica)(Mizutani, Sayama et al. 2011, Okazaki, Yasumoto et 80 al. 2016). Two related viruses have since been isolated from Taiwanese marbled eels (Anguilla 
81 marmorata) and a giant guitarfish (Rhynchobatus djiddensis)(Wen, Chen et al. 2015, Dill,

82 Camus et al. 2018). In contrast to the eel viruses, which encode S3H replicase proteins that

83 closely resemble the large tumor antigens (LT) of fish polyomaviruses, the guitarfish virus

84 encodes a distinct S3H replicase, called EO1, that is distant from LT and is instead more closely

85 related to the E1 replicases of papillomaviruses. The name "adomaviruses" has been applied to

86 this emerging family, connoting the fact that the three known species each encode homologs of

87 the adenain virion-maturational proteases of adenoviruses as well as polyomavirus and

88 papillomavirus $\mathrm{S} 3 \mathrm{H}$ homologs.

89 The primary goal of this study is to identify the adomavirus virion proteins and to uncover

90 possible evolutionary relationships to the virion proteins of small DNA tumor viruses. To

91 discover additional adomavirus species, we conducted metagenomic sequencing studies and

92 developed a pipeline to detect small DNA tumor virus-related sequences in the NCBI Sequence

93 Read Archive (SRA). Bioinformatic methods were used to predict which adomavirus ORFs

94 might represent virion proteins and the predictions were confirmed through functional expression

95 in cell culture. The results pave the way toward development of preventive vaccines against

96 pathogenic adomaviruses.

\section{Results}

\section{Detection of additional adomaviruses}

100 A post-mortem metagenomics analysis of an aquarium-bred Amazon red discus cichlid 101 (Symphysodon discus) exhibiting lethargy and inflamed skin lesions revealed a complete circular 102 adomavirus genome (Figure 2). Histopathological analysis of skin lesions from the discus 103 specimen showed no evidence of intranuclear inclusions or other obvious histopathology. 

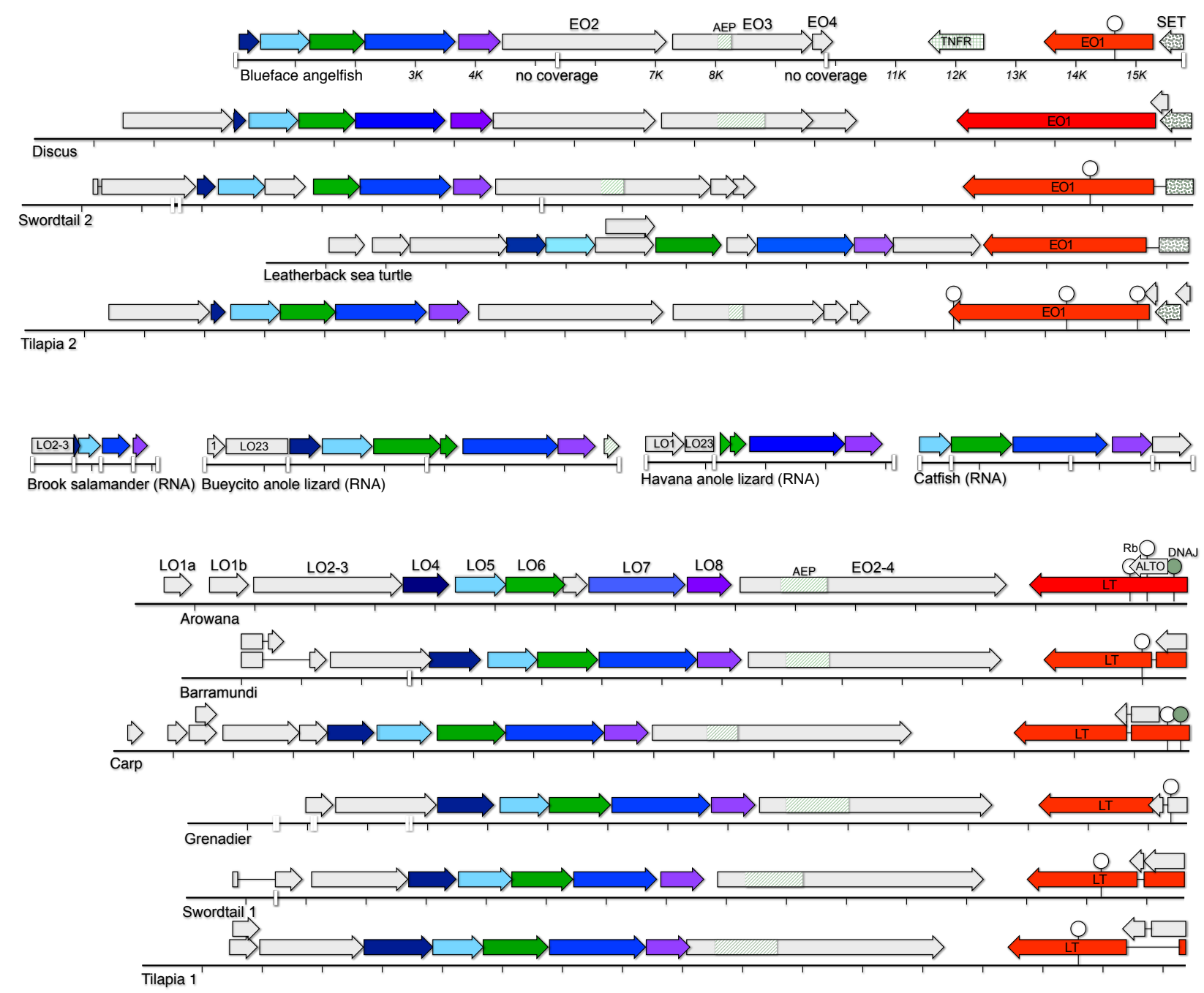

Figure 2: Genome maps of previously unknown adomaviruses. Genes were named based on conventions originally developed for Japanese eel adomavirus (NC_015123). Alpha adomaviruses (top half of figure) are defined by the presence of an adomavirusspecific $\mathrm{S} 3 \mathrm{H}$ replicase, designated EO1. A row of fragmentary adomavirus sequences from RNA datasets are shown in the middle of the figure. Beta adomaviruses (bottom half of figure) encode a polyomavirus-like large tumor antigen (LT). In some cases, repetitive or GC-rich patches (particularly near the 3' end of the LO2-3 ORF) could not be resolved using available short-read datasets. Coverage gaps are represented as white bars on the ruler. A poorly conserved set of accessory genes upstream of Alpha adomavirus EO1 genes show varying degrees of similarity to the S-adenosyl methionine-binding pocket of cellular SET proteins, which function as histone lysine-methyltransferases. Adomavirus SET homologs are highly divergent from all previously described eukaryotic and viral SET genes. The same is true for adomavirus EO2-4 and EO3 segments that encode homologs of the catalytic small subunit of archaeal eukaryotic primases (AEPs, hatched boxes).

Figure supplement 1: a table of accession numbers and Linnaean designations of hosts Figure supplement 2: examples of the annotation process 
bioRxiv preprint doi: https://doi.org/10.1101/341131: this version posted March 4 2020. The copyright holder for this preprint (which was not certified by peer review) is the author/funder. This article is a US Government work. It is not subject to copyright under 17 USC 105 and is also made available for use under a CCO license.

Figure supplement 3: GenBank-format nucleotide maps of adomaviruses

Figure supplement 4: protein compilations in fasta format

Figure supplement 5: splicing of marbled eel adomavirus transcripts

124

125

126

127

128

129

130

131

132

133

134

135

136

137

138

139

140

141

142

143

144

145

146

147

148

149

150

151

152

153
An adomavirus from an apparently healthy green arowana (Scleropages formosus) was first identified in TBLASTN searches of the NCBI Whole-Genome Shotgun (WGS) database as a set of short contigs with similarity to Japanese eel adomavirus proteins. A complete adomavirus genome was characterized by Sanger sequencing of overlapping PCR products using DNA left over from the original fin snip used for the WGS project (Bian, Hu et al. 2016).

The WGS TBLASTN survey also revealed a $4 \mathrm{~kb}$ contig with a sequence resembling adomavirus LT in a dataset for western softhead grenadier fish (Malacocephalus occidentalis) and a nearly complete adomavirus genome in a dataset for a skin biopsy of a leatherback sea turtle (Dermochelys coriacea). Genome sequences for the two viruses were completed using parent SRA datasets.

A pipeline using DIAMOND (a faster alternative to BLASTX (Buchfink, Xie et al. 2015)) was developed to screen SRA datasets for fish, amphibians, and reptiles. SRA datasets rich in reads encoding adomavirus-like protein sequences were subjected to de novo assembly. This approach resulted in the identification of seven additional adomavirus genomes (Figure 2). Notably, adomavirus sequences were found in genome sequencing datasets for farmed tilapia (Oreochromis niloticus), which represent a $\$ 7.5$ billion per year global aquaculture industry, and in the most extensively aquacultured fish in developing countries, the mirror carp (Cyprinus carpio)(Bacharach, Mishra et al. 2016, Belton, Little et al. 2018). Adomavirus-like fragments were also detected in transcriptomic datasets for brook salamander (Calotriton asper) and two closely related species of anole lizard (genus Anolis).

The WGS search for adomavirus-like $\mathrm{S} 3 \mathrm{H}$ sequences also led to the discovery of a divergent class of circular DNA elements that we tentatively designate "xenomaviruses," connoting the exotic nature of their predicted $\mathrm{S} 3 \mathrm{H}$ and virion proteins (Figure 2 Figure supplement 2, Figure 3, and Figure 4). Intriguingly, a conserved xenomavirus ORF shows distant predicted structural similarity to the L1 and VP1 penton proteins of papillomaviruses and polyomaviruses. The inferred replicase gene of a partial xenomavirus sequence detected in a dataset for pink abalone (Haliotis corrugata) encodes a domain with predicted structural similarity to the nickase domain of porcine circovirus 2 (a CRESS virus). The identification of additional viruses in this class could shed light on the evolutionary interrelationships between small DNA tumor viruses and CRESS viruses. 


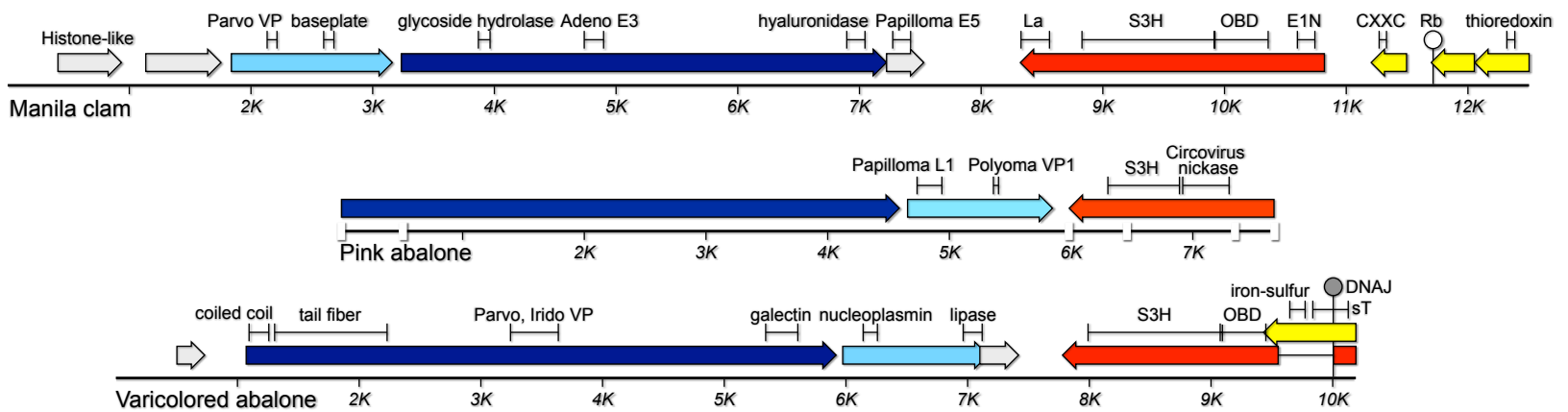

Figure 3: Genome maps of candidate xenomaviruses. Brackets indicate segments where remote similarities were detected in DELTA-BLAST, HHpred, and Phyre ${ }^{2}$ searches. The

\section{Adomavirus phylogeny}

Adomavirus sequences can be divided into two groups based on their replicative $\mathrm{S} 3 \mathrm{H}$ genes. A group we designate Alpha is defined by the presence of an EO1 S3H replicase gene that yields moderate hits (BLASTP E-values 1e-9) for papillomavirus E1 proteins. Adomavirus group Beta is defined by the presence of an $\mathrm{S} 3 \mathrm{H}$ replicase similar (E-values $\sim 1 \mathrm{e}-60$ ) to the LT proteins of polyomaviruses. A network display of BLASTP relationships is shown in Figure 4. The Alpha and Beta groupings are recapitulated in phylogenetic analyses of adomavirus LO8 (Adenain) homologs (Figure 4 Figure supplement 2).

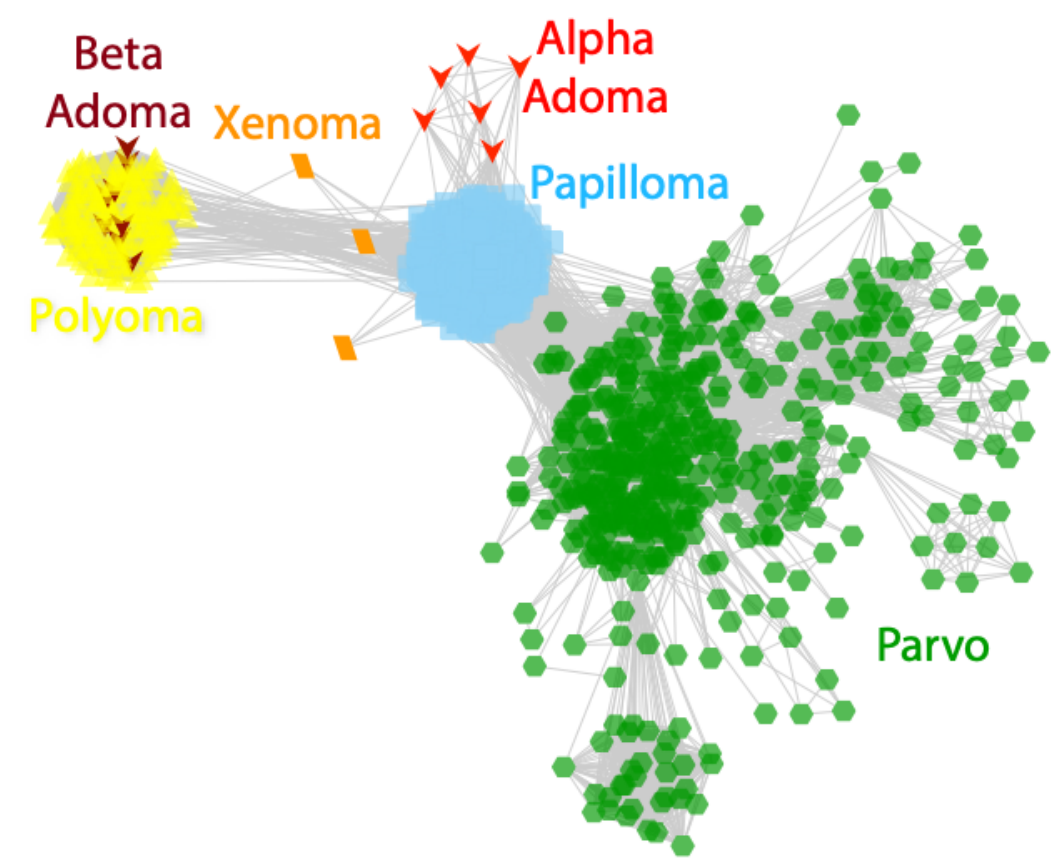


Figure supplement 1: an interactive version of the figure that can be viewed using Cytoscape software https://cytoscape.org

The adomavirus LT-like proteins share a full range of familiar polyomavirus-like features, including an N-terminal DnaJ domain, a potential retinoblastoma-interaction motif (LXCXE or LXXLFD)(An, Saenz Robles et al. 2012, Gouw, Michael et al. 2018). Several Alpha adomavirus EO1 proteins encode a C-terminal domain with predicted similarity to DNA-binding RFX-type winged helices (pfam02257). The RFX-like domain is conserved at the C-terminus of S3H proteins found in larger dsDNA (for example, vaccinia virus D5 YP232992), some parvoviruses (for example, bovine parvovirus NS1 NP_041402), and xenomaviruses. HHpred analyses indicate that, like other small DNA tumor virus replicases, both classes of adomavirus S3H proteins encode a central nicking endonuclease-like origin binding domain (Hickman, Ronning et al. 2002, Iyer, Koonin et al. 2005, Koonin, Dolja et al. 2015, Kazlauskas, Varsani et al. 2019).

\section{Bioinformatic prediction of adomavirus virion proteins}

To determine whether predicted adomavirus LO proteins are expressed from spliced or unspliced ORFs, RNAseq data published by Wen and colleagues (Wen, Chen et al. 2015) were analyzed to determine the splicing patterns of marbled eel adomavirus transcripts. Splice acceptors immediately upstream of the inferred ATG initiator codons of LO4, LO5, LO6, and LO7 proteins were extensively utilized (Figure 5 Figure supplement 5). Messenger RNAs encoding the adenovirus late genes carry a tripartite leader (TPL) that has been shown to enhance translation late in the adenovirus life cycle (Logan and Shenk 1984). A similar three-exon leader sequence was detected upstream of the marbled eel adomavirus LO genes (Figure 1).

HHpred searches were performed to detect remote similarities between the predicted structures of adomavirus proteins and known protein structures. Adomavirus LO4 proteins have a predicted $\mathrm{C}$-terminal trimeric coiled-coil domain. This relatively generic predicted fold gives a large and diverse range of hits in HHpred searches, including the coiled-coil domains of various viral fiber proteins (for example, avian reovirus $\sigma \mathrm{C}, 97 \%$ ). Negative-stain EM images indicate that adomavirus virions do not have a vertex fiber (Mizutani, Sayama et al. 2011, Wen, Chen et al. 2015, Dill, Camus et al. 2018). Intriguingly, LO4 proteins yielded low probability ( 50\%) HHpred hits for adenovirus pIX, a trimeric coiled coil protein that serves as a "cement" that smooths the triangular facets of the adenovirus virion. The hypothesis that LO4 is a pIX homolog could hypothetically account for the smooth appearance of adomavirus virion facets in negativestain EM.

A C-terminal segment of some LO5 sequences, as well as alignments of multiple LO5 sequences, yielded moderate ( $\sim 60 \%$ probability) HHpred hits for CvsA1_340L protein (singlejellyroll vertex penton) of Paramecium bursaria chlorella virus 1 (PDB:6 $\overline{N C L}$ a6). A screen

206 shot of a typical HHpred result is shown in Figure 2 Figure supplement 2.

207 Alignments of LO6 ORFs show high-probability (95\%) HHPred hits for a 37 amino acid 
211 believed to play a role in destabilizing cellular membranes during the infectious entry process

212 (Moyer, Besser et al. 2015). The results suggest that LO6 might be a fused homolog of

213 adenovirus $\mathrm{pVI}$ and $\mathrm{pX}$ virion core proteins.

214 HHpred searches using LO7 sequences did not produce interpretable results, with the single

215 exception of the LO7 sequence of grenadier adomavirus, which gives a moderate-probability hit

216 for the V20 double-jellyroll hexon major capsid protein of Sputnik virophage (Figure 2 Figure

217 supplement 2).

218 In addition to offering a convenient way to summarize aggregate BLASTP interrelationships

219 (e.g., Figure 4), all-against-all sequence similarity network analysis can be a useful method for

220 discovering distant similarities between highly divergent groups of proteins (Iranzo, Krupovic et

221 al. 2017). In one noteworthy example, network analyses were recently used to detect remote

222 sequence similarities between small DNA tumor virus S3H replicases and CRESS virus

223 replicases (Kazlauskas, Varsani et al. 2019). In contrast to traditional analyses using

224 phylogenetic trees, it is possible for network analyses to detect individual pairs of sequences in

225 separate clusters that both happen to have preserved the primary sequence of a common ancestor.

226 We performed low-stringency network analyses to further investigate possible remote sequence

227 similarities between adenovirus, adintovirus, and candidate adomavirus virion proteins.

228 Networks for adomavirus LO4 (inferred fiber or cement protein) and LO7 (inferred double-

229 jellyroll hexon major capsid protein) showed few or no connections to adenovirus or adintovirus

230 virion protein sequences, even at a BLASTP E-value threshold of 1e-1. In contrast, LO8

231 (adenain) proteins yielded informative networks at E-value thresholds of 1e-5 (Figure 5). At less

232 stringent E-value thresholds (1e-2) similarities between adomavirus LO5 (inferred single-

233 jellyroll vertex penton) and inferred adintovirus penton protein sequences emerged. PSI-BLAST

234 searches using LO5 alignments confirmed the apparent sequence similarities (E-values $\sim 1 \mathrm{e}-6)$ to

235 predicted adintovirus penton proteins found in arthropod and coral datasets (e.g., GBM63801

236 EFA12278 LSMT01002030). Although adenovirus pVI did not cluster with LO6 (inferred virion

237 core protein) or proposed adintovirus virion core proteins (Cupiennin, GasderminX, PLA2X) at

238 an E-value threshold of 1e-2, Alpha adomavirus LO6 proteins clustered with adintovirus

239 cupiennin and Beta adomavirus LO6 proteins clustered with adenovirus pX proteins. 


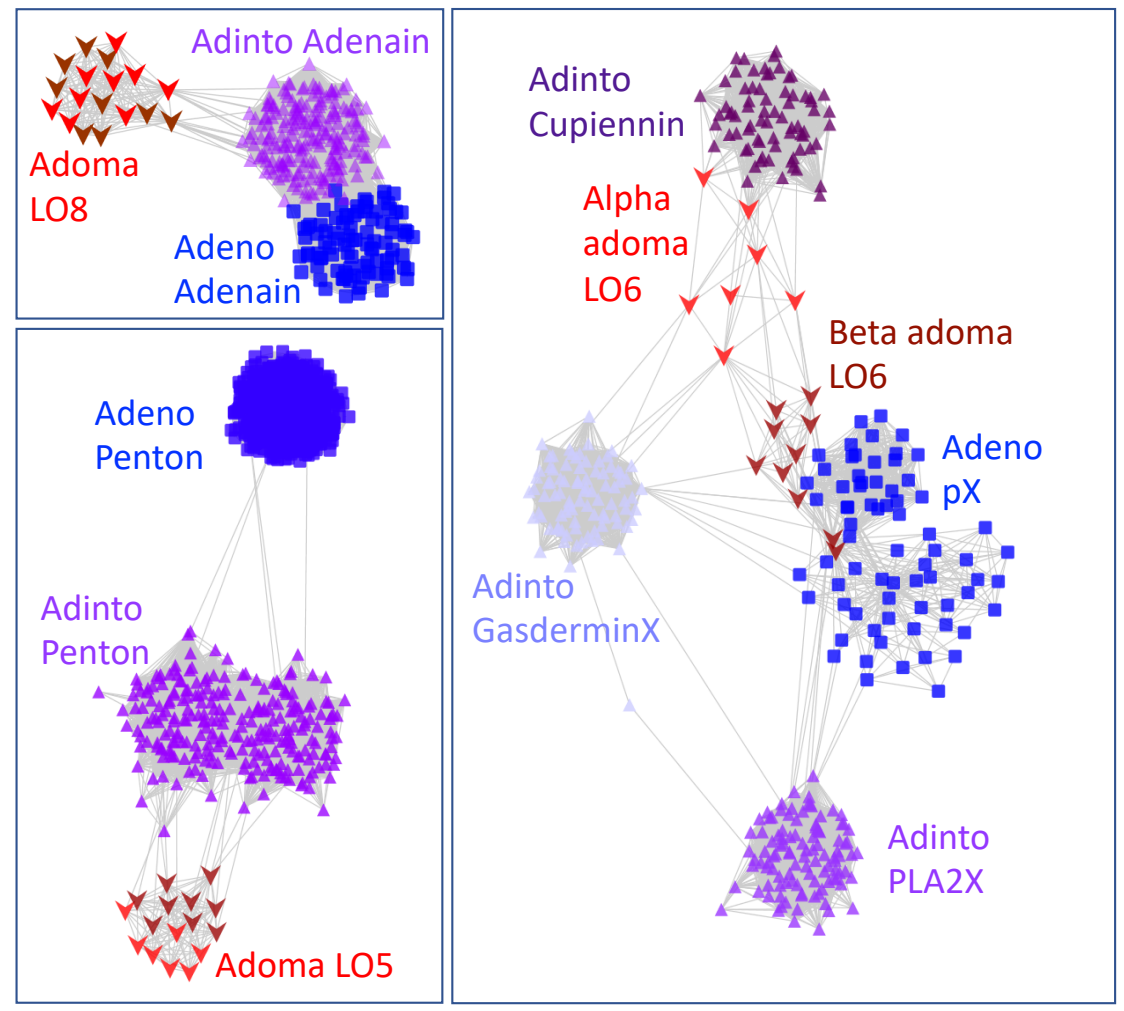

241 Figure 5: Sequence similarity network analysis of predicted minor virion proteins. The Adenain (LO8) network was constructed with a BLASTP E-value threshold of 1e-5. Penton (LO5) and virion core protein (LO6) networks used a threshold of 1e-2.

244 Figure supplement 1: an interactive version of the figure that can be viewed using Cytoscape software https://cytoscape.org

\section{Experimental confirmation of predicted adomavirus virion proteins}

The bioinformatic results suggest that the LO4-8 operon encodes syntenic homologs of adenovirus and adintovirus virion proteins. To experimentally test this prediction, marbled eel adomavirus was grown in eel kidney cell culture (Wen, Chen et al. 2015) and virions were purified using Optiprep gradient ultracentrifugation. Virion-enriched gradient fractions were separated on SDS-PAGE gels and bands were subjected to mass spectrometric analysis (Figure 6). The analysis identified prominent bands in the Coomassie-stained gel as LO4, LO5, LO7, and LO8. The relative intensities of bands identified as LO5 and LO7 are consistent with the prediction that the two proteins constitute penton and hexon subunits, respectively. Lower molecular weight bands showed hits for LO6, suggesting that this protein is present in virions in an LO8 (adenain) cleaved form. LO6 proteins were found to encode potential adenain cleavage motifs ((MIL)XGXG or L(LR)GG) (Ruzindana-Umunyana, Imbeault et al. 2002). A list of protein modifications observed in the mass spectrometric analysis is shown in Figure 6 Figure 260 supplement 1. 


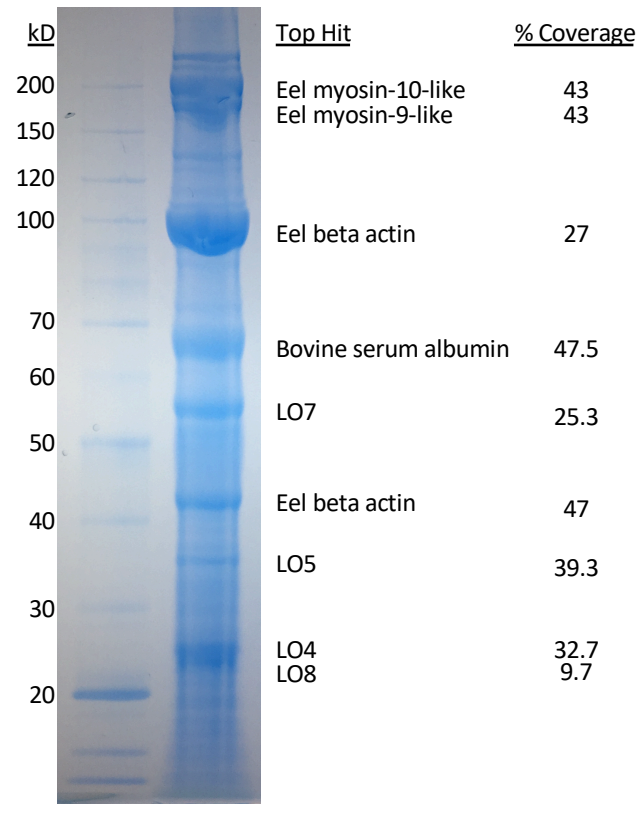

\section{Expression of recombinant virion proteins} a Q Exactive HF Mass Spectrometer. results

Figure 6: SDS-PAGE analysis of marbled eel adomavirus virions purified from infected EK-1 cells, with bands annotated by mass spectrometric analysis. Clarified lysates of infected cells were ultracentrifuged through an Optiprep gradient. Peak virioncontaining fractions were selected then subjected to size exclusion chromatography over agarose resin. The sample was then subjected to TCA precipitation and run on an SDSPAGE gel. Thirteen gel bands were individually excised, trypsin-digested, and analyzed on

Figure supplement 1: post-translational modifications observed in mass spectrometric

Adenovirus penton proteins can spontaneously assemble into 12-pentamer subviral particles that may serve as decoy pseudocapsids in vivo (Vragniau, Hubner et al. 2017). Similarly, recombinant polyomavirus and papillomavirus penton proteins can spontaneously assemble into icosahedral virus-like particles (VLPs) that closely resemble native virions. We are not aware of any reports of production of full-size (i.e., hexon+penton) adenovirus VLPs. To investigate the behavior of recombinant adomavirus virion proteins, codon-modified marbled eel adomavirus LO1-LO8 expression plasmids were transfected individually into human 293TT cells (Buck, Pastrana et al. 2004). Optiprep ultracentrifugation was used to separate virus-like particles (VLPs) from smaller solutes. A human papillomavirus type 16 (HPV16) L1/L2 expression plasmid was used as a positive control for VLP formation (Buck, Thompson et al. 2005). Cells transfected with adomavirus LO4, LO5, or LO7 expression constructs each produced particles that migrated into the core fractions of Optiprep gradients, whereas cells transfected with LO1, 
LO2-3, LO6, or LO8 alone did not show evidence of particle formation (Figure 7). Negativestain EM analysis showed that the LO4, LO5, and LO7 particles were irregular (Figure 7 Figure supplement 1). In co-transfections of various combinations of LO genes, it was found that inclusion of LO6 inhibited the formation of LO5 and LO7 particles but did not impair the formation of LO4 particles (Figure 8). The fact that over-expression of LO6 can antagonize particle formation supports the bioinformatic prediction that LO6 is a minor virion component

292 that directly interacts with LO5 and LO7.

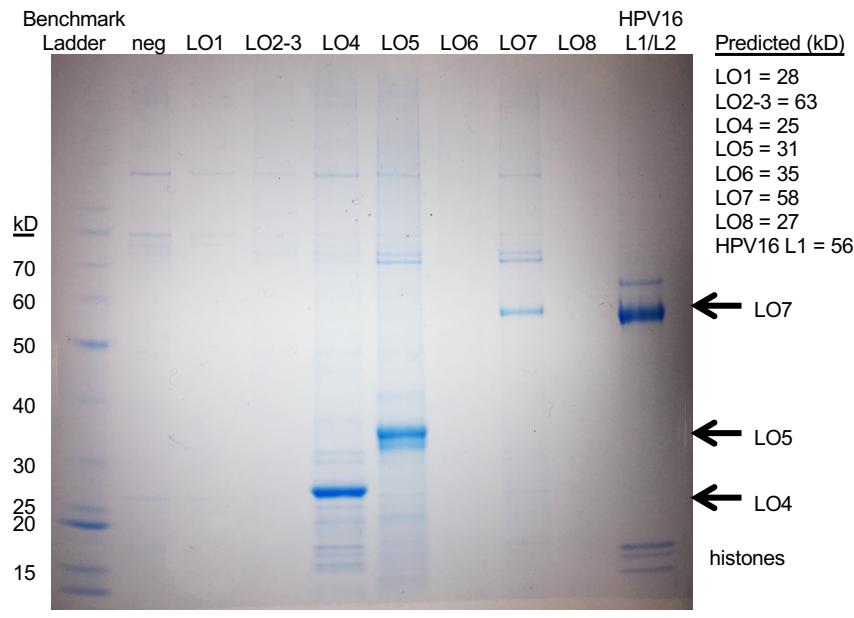

Figure 7: SDS-PAGE analysis VLPs assembled in cells transfected with individual LO expression constructs. 293TT cells were transfected with individual codon modified marbled eel LO expression plasmids indicated at the top of the image. The cells were lysed, subjected to nuclease digestion and a clarifying $5000 \mathrm{x} g$ spin. Soluble material was ultracentrifuged through Optiprep gradients. Core gradient fractions with peak VLP content were subjected to SDS-PAGE analysis.

Figure supplement 1: Negative-stain electron microscopy of recombinant particles. 


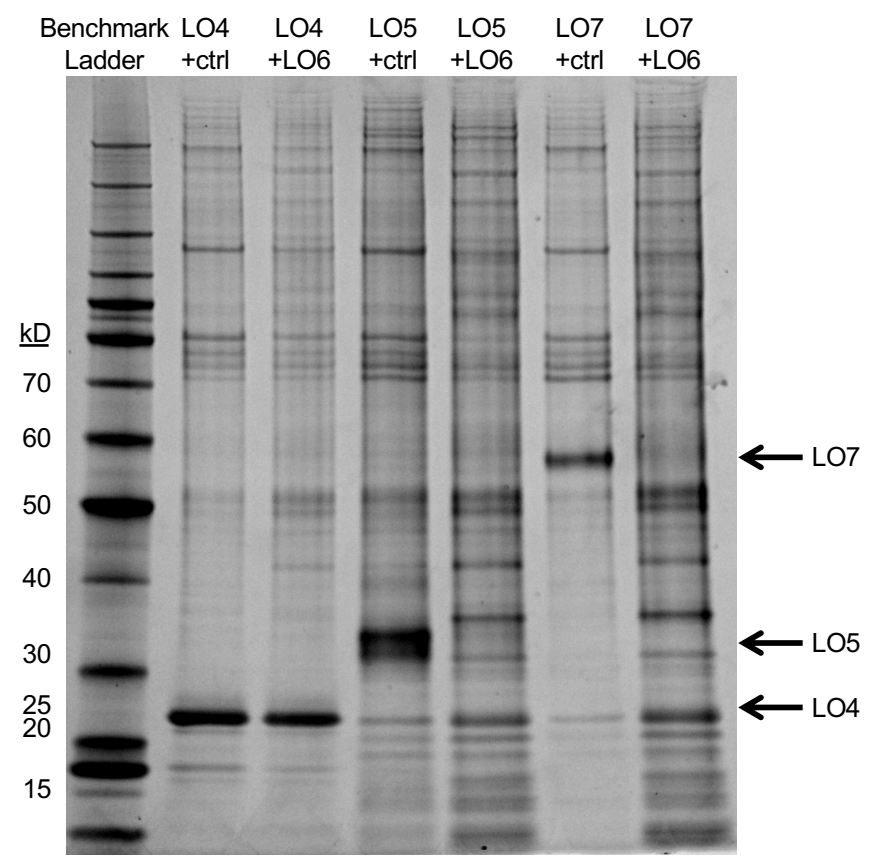
LO7 (but not LO4) particle formation. 293TT cells were transfected with the indicated combination of plasmids and subjected to ultracentrifugal separation through Optiprep gradients.

Individually expressed LO5 (penton) and LO7 (hexon) particle preparations showed dsDNA signal in Quant-iT PicoGreen assays (Invitrogen), indicating the presence of nuclease-resistant encapsidated DNA within the purified particles. Optiprep-purified particle preparations from cells co-transfected with LO4, LO5, and LO7 were subjected to an additional round of nuclease digestion with salt-tolerant Benzonase endonuclease (Sigma) followed by agarose gel filtration to remove the nuclease and any residual digested DNA fragments. Nuclease-treated/gel filtered particles typically contained roughly seven nanograms of DNA per microgram of total protein, confirming the presence of nuclease-resistant nonspecific cellular DNA within the particles. The observation is reminiscent of findings for recombinant papillomavirus VLPs (Buck, Thompson et

317 al. 2005).

\section{Discussion}

320 We have identified a dozen new representatives of the emerging virus family Adomaviridae.

321 Four of the new sequences are associated with terrestrial vertebrates, extending the known host range beyond fish. Phylogenetic analyses reveal two adomavirus lineages that appear to have independently co-evolved with host animals. This observation suggests that the two adomavirus lineages both infected the first jawed vertebrates roughly half a billion years ago. Within both adomavirus lineages, there are members associated with commercially important fish species. 
Vaccine immunogens comprised of recombinant VLPs have been highly successful in humans (Schiller and Lowy 2015). In particular, vaccines against HPVs have proven remarkably immunogenic, even after a single dose. Our identification of adomavirus virion proteins and demonstration of their ability to assemble into roughly spherical DNA-containing particles should facilitate the development of recombinant subunit vaccines against these viruses, some of which are known to cause severe disease in fish.

The adomavirus virion protein genes, penton (LO5), core (LO6), hexon (LO7), and adenain (LO8), appear to be syntenic homologs of adenovirus and adintovirus virion protein genes. The results also suggest that LO4 may be a homolog of adenovirus pIX, a trimeric coiled coil protein that cements the facets of the adenovirus virion. At a primary sequence level, adomavirus virion proteins more closely resemble adintovirus virion proteins, rather than adenovirus virion proteins (Figure 5). These results tie the Adomaviridae into a broad consortium of eukaryotic virus families (Koonin, Krupovic et al. 2015).

In unicellular eukaryotes, non-enveloped midsize (10-50 kb) dsDNA viruses have been shown to have a remarkable degree of genetic modularity (Koonin, Dolja et al. 2015, Yutin, Shevchenko et al. 2015) https://www.biorxiv.org/content/10.1101/697771v3. The pairing of related virion proteins in adenoviruses, adintoviruses, and adomaviruses with entirely different classes of DNA replicase genes thus has ample precedence in non-animal eukaryotes. It will be important to apply emerging higher-throughput search algorithms, such as Mash Screen (Ondov, Starrett et al. 2019) and Cenote-Taker (Tisza, Pastrana et al. 2019), to exhaustively search for each of the overlapping hallmark genes of this virus supergroup in genomic, transcriptomic, and metagenomic surveys, particularly datasets for terrestrial vertebrates.

\section{Sample Collection and cell culture}

A red discus cichlid (Symphysodon discus) was purchased at a pet shop in Gainesville, Florida. The fish was moribund and showed erythematous skin lesions. Propagation of the discus adomavirus in cell culture was attempted by overlaying skin tissue homogenates on Grunt Fin (GF) and Epithelioma Papulosum Cyprini cell lines (ATCC). Neither cytopathic effects nor qPCR-based detection of viral replication were observed during two blind passages of 14 days each.

358 Dr. Chiu-Ming Wen generously provided EK-1 cells (a Japanese eel kidney line) infected with the Taiwanese marbled eel adomavirus (Wen, Chen et al. 2015). The virus was propagated by inoculation of supernatants from the infected culture into uninfected EK-1 cells cultured at room temperature in DMEM with $10 \%$ fetal calf serum. Human embryonic kidney-derived 293TT cells were cultured as previously described (Buck, Pastrana et al. 2004). 


\section{Viral genome sequencing}

364 For the discus adomavirus, total DNA was extracted from a skin lesion and subjected to deep sequencing. Marbled eel adomavirus virions were purified from lysates of infected EK-1 cells using Optiprep gradient ultracentrifugation (Peretti, FitzGerald et al. 2015). DNA extracted from Optiprep gradient fractions was subjected to rolling circle amplification (RCA, TempliPhi, GE Health Sciences). The marbled eel adomavirus RCA products and discus total DNA were prepared with a Nextera XT DNA Sample Prep kit and sequenced using the MiSeq (Illumina) sequencing system with $2 \times 250$ bp paired-end sequencing reagents. In addition, the marbled eel adomavirus RCA product was digested with $A c l I$ and EcoRI restriction enzymes and the resulting early and late halves of the viral genome were cloned separately into the AclI and EcoRI restriction sites of pAsylum+. The sequence of the cloned genome was verified by a combination of MiSeq and Sanger sequencing. The clones are available upon request.

For the arowana adomavirus, overlapping PCR primers were designed based on WGS accession numbers LGSE01029406, LGSE01031009, LGSE01028643, LGSE01028176, and LGSE01030049 (Bian, Hu et al. 2016). PCR products were subjected to primer-walking Sanger 378 sequencing.

\section{Discovery of viral sequences in NCBI databases}

Papillomavirus E1 sequences were downloaded from PaVE https://pave.niaid.nih.gov (Van Doorslaer, Li et al. 2017). Polyomavirus LT sequences were downloaded from PyVE https://ccrod.cancer.gov/confluence/display/LCOTF/Polyomavirus (Buck, Van Doorslaer et al. 2016). Parvovirus NS1 proteins and S3H proteins of CRESS viruses and virophage-like viruses were compiled from multiple databases, including RefSeq, WGS, and TSA, using TBLASTN searches. Adenovirus, virophage, and bacteriophage PolB sequences were downloaded from

386 GenBank nr using DELTA-BLAST searches (Boratyn, Schaffer et al. 2012) with Alpha or Beta 387 adintovirus PolB proteins as bait.

SRA datasets for fish, amphibians, and reptiles were searched using DIAMOND (Buchfink, Xie et al. 2015) or NCBI SRA Toolkit (http://www.ncbi.nlm.nih.gov/books/NBK158900/) in TBLASTN mode using adomavirus protein sequences as the subject database or query, respectively. Reads with similarity to the baits were collected and subjected to BLASTX searches against a custom library of viral proteins representing adomaviruses and other small DNA tumor viruses. SRA datasets of interest were subjected to de novo assembly using the SPAdes suite (Bankevich, Nurk et al. 2012, Nurk, Meleshko et al. 2017) or Megahit (Li, Liu et al. 2015, Li, Luo et al. 2016). Contigs encoding virus-like proteins were identified by TBLASTN searches against adomavirus protein sequences using Bowtie (Langmead and Salzberg 2012). The candidate contigs were validated using the CLC Genomics Workbench 12 align to reference function.

Predicted protein sequences were automatically extracted from contigs of interest using getorf (http://bioinfo.nhri.org.tw/cgi-bin/emboss/getorf)(Rice, Longden et al. 2000). Sequences were clustered using EFI-EST (https://efi.igb.illinois.edu/efi-est/)(Gerlt, Bouvier et al. 2015, Zallot,

403 Multiple sequence alignments were constructed using MAFFT

404 (https://toolkit.tuebingen.mpg.de/\#/tools/mafft)(Kuraku, Zmasek et al. 2013, Katoh, Rozewicki 
et al. 2019). Individual or aligned protein sequences were subjected to HHpred searches (https://toolkit.tuebingen.mpg.de/\#/tools/hhpred)(Hildebrand, Remmert et al. 2009, Meier and Soding 2015, Zimmermann, Stephens et al. 2017) against PDB, Pfam-A, NCBI Conserved Domains, and PRK databases.

409 Contigs were annotated using Cenote-Taker (Tisza, Pastrana et al. 2019) with an iteratively refined library of conserved adintovirus protein sequences. Maps were drawn using MacVector 17. Phylogenetic analyses were performed using Phylogeny.fr with default settings (Dereeper, 412 Guignon et al. 2008).

\section{Marbled eel adomavirus transcript analysis, late ORF expression, and virion purification}

414 RNAseq reads reported by Wen et al (Wen, Chen et al. 2015) were aligned to the marbled eel 415 adomavirus genome using HISAT2 version 2.0.5 (Kim, Langmead et al. 2015) with the

416 following options: “--rna-strandness FR --dta --no-mixed --no-discordant". Integrated Genome Viewer (IGV) version 2.4.9 (Robinson, Thorvaldsdottir et al. 2017) was used to determine splice junctions and their depth of coverage. Additional validation was performed by visual inspection using CLC Genomics Workbench 12.

420 Codon-modified expression constructs encoding the marbled eel adomavirus LO1-LO8 proteins (https://github.com/BUCK-LCO-NCI/Codmod as_different as possible)(Pastrana, Buck et al. 2004). 293TT cells were transfected with LO expression constructs for roughly 48 hours. Cells were lysed in a small volume of PBS with $0.5 \%$ Triton X-100 or Brij-58 and Benzonase Dnase/Rnase (Sigma)(Buck and Thompson 2007). After one hour of maturation at neutral $\mathrm{pH}$, the lysate was clarified at $5000 \mathrm{x}$ g for $10 \mathrm{~min}$. The clarified lysate was loaded onto a 15-27-33$39-46 \%$ Optiprep gradient in PBS with $0.8 \mathrm{M} \mathrm{NaCl}$. Gradient fractions were collected by bottom puncture of the tube and screened by PicoGreen dsDNA stain (Invitrogen), BCA, or SDS-PAGE analysis. Electron microscopic analysis was performed by spotting several microliters of Optiprep fraction material (or, in some instances, particles exchanged out of Optiprep using agarose gel filtration) onto carbon film copper grids, followed by staining with $0.5 \%$ uranyl acetate.

\section{Mass Spectrometry}

Optiprep-purified marbled eel adomavirus virions were precipitated with trichloroacetic acid. A $1 \mathrm{ml}$ sample was treated with $100 \mu \mathrm{l}$ of $0.15 \%$ deoxycholic acid and incubated at room temperature for 10 minutes. $100 \mu \mathrm{l}$ of $100 \%$ TCA was then added and the sample was vortexed and incubated on ice for 30 minutes. Following the incubation, the sample was centrifuged at $10,000 \mathrm{x}$ g for 10 minutes at $4^{\circ} \mathrm{C}$. The supernatant was removed, and the remaining pellet was washed with ice-cold acetone to remove residual TCA. The protein pellet was solublized with NuPAGE Sample Buffer $+5 \%$ BME (Sigma) and run on a 10-12\% Bis-Tris MOPS gel (Thermo). The protein bands were visualized using InstantBlue (Expedeon). Thirteen gel bands were individually excised and placed into $1.5 \mathrm{ml}$ Eppendorf tubes. The gel bands were sent to the National Cancer Institute in Fredrick, Maryland where they were de-stained, digested with trypsin, and processed on a Thermo Fisher Q Exactive HF Mass Spectrometer. Thermo Proteome Discoverer 2.2 software was used for initial protein identification. The uninterpreted mass spectral data were also searched against Anguilla proteins (Swiss-Prot and TrEMBL database 
containing 105,268 proteins), Bos taurus proteins (Swiss-Prot and TrEMBL database containing 48,288 proteins), a common contaminants database (cRAPome), and translated marbled eel adomavirus ORFs. Further analysis was conducted using Protein Metrics Biopharma software to identify modifications missed in initial analyses.

\section{Ethics Statement}

All animal tissue samples were received as diagnostic specimens collected for pathogen testing and disease investigation purposes.

\section{Data Availability}

456 GenBank accession numbers for sequences deposited in association with this study are

457 BK010891 BK010892 BK011012 BK011013 BK011014 BK011015 BK011016 BK011017 MF946550 MH282863.

\section{Acknowledgments}

The authors are indebted to Eugene Koonin and Natalya Yutin for their generous guidance and for the spirited discussions that inspired us to pursue this study. We are particularly grateful to them for sharing their observation that adomaviruses encode a recognizable adenain homolog and their discovery of adomavirus sequences in the arowana WGS datasets. The authors are also grateful to Lisa Jenkins for her extensive technical guidance on analyzing mass spectrometric data. We thank Karl Münger for useful discussions, including advice about oncogene sequence motifs.

\section{References}

An, P., M. T. Saenz Robles and J. M. Pipas (2012). "Large T antigens of polyomaviruses: amazing molecular machines." Annu Rev Microbiol 66: 213-236. Orthomyxo-like Virus Causing Mass Die-Offs of Tilapia." mBio 7(2): e00431-00416. Bankevich, A., S. Nurk, D. Antipov, A. A. Gurevich, M. Dvorkin, A. S. Kulikov, V. M. Lesin, S. I. Nikolenko, S. Pham, A. D. Prjibelski, A. V. Pyshkin, A. V. Sirotkin, N. Vyahhi, G. Tesler, M. A. Alekseyev and P. A. Pevzner (2012). "SPAdes: a new genome assembly algorithm and its applications to single-cell sequencing." J Comput Biol 19(5): 455-477.

Belton, B., D. Little and S. Bush. (2018). "Let them eat carp: Fish farms are helping to fight hunger." from https://theconversation.com/let-them-eat-carp-fish-farms-are-helping-to-fighthunger-90421.

Bian, C., Y. Hu, V. Ravi, I. S. Kuznetsova, X. Shen, X. Mu, Y. Sun, X. You, J. Li, X. Li, Y. Qiu, B. H. Tay, N. M. Thevasagayam, A. S. Komissarov, V. Trifonov, M. Kabilov, A. Tupikin, J. 

Zhang, Z. Xiong, Z. Tang, C. Peng, Z. Ruan, H. Yu, J. Chen, M. Fan, Y. Huang, M. Wang, X. Zhao, G. Hu, H. Yang, J. Wang, J. Wang, X. Xu, L. Song, G. Xu, P. Xu, J. Xu, S. J. O'Brien, L. Orban, B. Venkatesh and Q. Shi (2016). "The Asian arowana (Scleropages formosus) genome provides new insights into the evolution of an early lineage of teleosts." Sci Rep 6: 24501. Boratyn, G. M., A. A. Schaffer, R. Agarwala, S. F. Altschul, D. J. Lipman and T. L. Madden (2012). "Domain enhanced lookup time accelerated BLAST." Biol Direct 7: 12. Buchfink, B., C. Xie and D. H. Huson (2015). "Fast and sensitive protein alignment using DIAMOND." Nat Methods 12(1): 59-60.

Buck, C. B., D. V. Pastrana, D. R. Lowy and J. T. Schiller (2004). "Efficient intracellular assembly of papillomaviral vectors." J Virol 78(2): 751-757. Buck, C. B. and C. D. Thompson (2007). "Production of papillomavirus-based gene transfer vectors." Curr Protoc Cell Biol Chapter 26: Unit 26.21.

501 Buck, C. B., C. D. Thompson, Y. Y. Pang, D. R. Lowy and J. T. Schiller (2005). "Maturation of papillomavirus capsids." J Virol 79(5): 2839-2846. Buck, C. B., K. Van Doorslaer, A. Peretti, E. M. Geoghegan, M. J. Tisza, P. An, J. P. Katz, J. M. Pipas, A. A. McBride, A. C. Camus, A. J. McDermott, J. A. Dill, E. Delwart, T. F. Ng, K. Ancient Evolutionary History of Polyomaviruses." PLoS Pathog 12(4): e1005574. de Souza, R. F., L. M. Iyer and L. Aravind (2010). "Diversity and evolution of chromatin proteins encoded by DNA viruses." Biochim Biophys Acta 1799(3-4): 302-318. Dereeper, A., V. Guignon, G. Blanc, S. Audic, S. Buffet, F. Chevenet, J. F. Dufayard, S. Guindon, V. Lefort, M. Lescot, J. M. Claverie and O. Gascuel (2008). "Phylogeny.fr: robust phylogenetic analysis for the non-specialist." Nucleic Acids Res 36(Web Server issue): W465469. Evidence of the First Elasmobranch Adomavirus, the Cause of Skin Disease in a Giant Guitarfish, Rhynchobatus djiddensis." MBio 9(3). Whalen (2015). "Enzyme Function Initiative-Enzyme Similarity Tool (EFI-EST): A web tool for generating protein sequence similarity networks." Biochim Biophys Acta 1854(8): 1019-1037. Gouw, M., S. Michael, H. Samano-Sanchez, M. Kumar, A. Zeke, B. Lang, B. Bely, L. B. Chemes, N. E. Davey, Z. Deng, F. Diella, C. M. Gurth, A. K. Huber, S. Kleinsorg, L. S. Schlegel, N. Palopoli, K. V. Roey, B. Altenberg, A. Remenyi, H. Dinkel and T. J. Gibson (2018). "The eukaryotic linear motif resource - 2018 update." Nucleic Acids Res 46(D1): D428-D434. Hickman, A. B., D. R. Ronning, R. M. Kotin and F. Dyda (2002). "Structural unity among viral origin binding proteins: crystal structure of the nuclease domain of adeno-associated virus Rep." Mol Cell 10(2): 327-337.

Hildebrand, A., M. Remmert, A. Biegert and J. Soding (2009). "Fast and accurate automatic structure prediction with HHpred." Proteins 77 Suppl 9: 128-132.

Iranzo, J., M. Krupovic and E. V. Koonin (2017). "A network perspective on the virus world." Commun Integr Biol 10(2): e1296614.

530 Iyer, L. M., E. V. Koonin, D. D. Leipe and L. Aravind (2005). "Origin and evolution of the 531 archaeo-eukaryotic primase superfamily and related palm-domain proteins: structural insights 532 and new members." Nucleic Acids Res 33(12): 3875-3896. 
Katoh, K., J. Rozewicki and K. D. Yamada (2019). "MAFFT online service: multiple sequence alignment, interactive sequence choice and visualization." Brief Bioinform 20(4): 1160-1166. Kazlauskas, D., A. Varsani, E. V. Koonin and M. Krupovic (2019). "Multiple origins of prokaryotic and eukaryotic single-stranded DNA viruses from bacterial and archaeal plasmids." Nat Commun 10(1): 3425.

538 Kim, D., B. Langmead and S. L. Salzberg (2015). "HISAT: a fast spliced aligner with low memory requirements." Nat Methods 12(4): 357-360. Koonin, E. V., V. V. Dolja and M. Krupovic (2015). "Origins and evolution of viruses of eukaryotes: The ultimate modularity." Virology 479-480: 2-25.

Koonin, E. V., M. Krupovic and N. Yutin (2015). "Evolution of double-stranded DNA viruses of eukaryotes: from bacteriophages to transposons to giant viruses." Ann N Y Acad Sci 1341: 1024.

Krupovic, M. and E. V. Koonin (2017). "Multiple origins of viral capsid proteins from cellular ancestors." Proc Natl Acad Sci U S A 114(12): E2401-E2410.

Kuraku, S., C. M. Zmasek, O. Nishimura and K. Katoh (2013). "aLeaves facilitates on-demand exploration of metazoan gene family trees on MAFFT sequence alignment server with enhanced interactivity." Nucleic Acids Res 41(Web Server issue): W22-28. Langmead, B. and S. L. Salzberg (2012). "Fast gapped-read alignment with Bowtie 2." Nat Methods 9(4): 357-359.

552 Li, D., C. M. Liu, R. Luo, K. Sadakane and T. W. Lam (2015). "MEGAHIT: an ultra-fast singlenode solution for large and complex metagenomics assembly via succinct de Bruijn graph." Bioinformatics 31(10): 1674-1676. Li, D., R. Luo, C. M. Liu, C. M. Leung, H. F. Ting, K. Sadakane, H. Yamashita and T. W. Lam (2016). "MEGAHIT v1.0: A fast and scalable metagenome assembler driven by advanced methodologies and community practices." Methods 102: 3-11.

558 Logan, J. and T. Shenk (1984). "Adenovirus tripartite leader sequence enhances translation of mRNAs late after infection." Proc Natl Acad Sci U S A 81(12): 3655-3659. Meier, A. and J. Soding (2015). "Automatic Prediction of Protein 3D Structures by Probabilistic Multi-template Homology Modeling." PLoS Comput Biol 11(10): e1004343. Mizutani, T., Y. Sayama, A. Nakanishi, H. Ochiai, K. Sakai, K. Wakabayashi, N. Tanaka, E. Miura, M. Oba, I. Kurane, M. Saijo, S. Morikawa and S. Ono (2011). "Novel DNA virus isolated from samples showing endothelial cell necrosis in the Japanese eel, Anguilla japonica." Virology 412(1): 179-187.

566 Moyer, C. L., E. S. Besser and G. R. Nemerow (2015). "A Single Maturation Cleavage Site in 567 Adenovirus Impacts Cell Entry and Capsid Assembly." J Virol 90(1): 521-532.

568 Nemerow, G. R., P. L. Stewart and V. S. Reddy (2012). "Structure of human adenovirus." Curr 569 Opin Virol 2(2): 115-121.

570 Nurk, S., D. Meleshko, A. Korobeynikov and P. A. Pevzner (2017). "metaSPAdes: a new

571 versatile metagenomic assembler." Genome Res 27(5): 824-834.

572 Okazaki, S., S. Yasumoto, S. Koyama, S. Tsuchiaka, Y. Naoi, T. Omatsu, S. Ono and T.

573 Mizutani (2016). "Detection of Japanese eel endothelial cells-infecting virus in Anguilla japonica 574 elvers." J Vet Med Sci 78(4): 705-707.

575 Ondov, B. D., G. J. Starrett, A. Sappington, A. Kostic, S. Koren, C. B. Buck and A. M. Phillippy 576 (2019). "Mash Screen: high-throughput sequence containment estimation for genome discovery." 577 Genome Biol 20(1): 232. 
578 Pastrana, D. V., C. B. Buck, Y. Y. Pang, C. D. Thompson, P. E. Castle, P. C. FitzGerald, S.

579 Kruger Kjaer, D. R. Lowy and J. T. Schiller (2004). "Reactivity of human sera in a sensitive,

580 high-throughput pseudovirus-based papillomavirus neutralization assay for HPV16 and HPV18."

581 Virology 321(2): 205-216.

582 Peretti, A., P. C. FitzGerald, V. Bliskovsky, C. B. Buck and D. V. Pastrana (2015). "Hamburger

583 polyomaviruses." J Gen Virol 96(Pt 4): 833-839.

584 Pipas, J. M. (2019). "DNA Tumor Viruses and Their Contributions to Molecular Biology." J

585 Virol 93(9).

586 Rice, P., I. Longden and A. Bleasby (2000). "EMBOSS: the European Molecular Biology Open

587 Software Suite." Trends Genet 16(6): 276-277.

588 Robinson, J. T., H. Thorvaldsdottir, A. M. Wenger, A. Zehir and J. P. Mesirov (2017). "Variant

589 Review with the Integrative Genomics Viewer." Cancer Res 77(21): e31-e34.

590 Ruzindana-Umunyana, A., L. Imbeault and J. M. Weber (2002). "Substrate specificity of

591 adenovirus protease." Virus Res 89(1): 41-52.

592 Schiller, J. T. and D. R. Lowy (2015). "Raising Expectations For Subunit Vaccine." J Infect Dis

593 211(9): 1373-1375.

594 Shannon, P., A. Markiel, O. Ozier, N. S. Baliga, J. T. Wang, D. Ramage, N. Amin, B.

595 Schwikowski and T. Ideker (2003). "Cytoscape: a software environment for integrated models of biomolecular interaction networks." Genome Res 13(11): 2498-2504.

Tisza, M. J., D. V. Pastrana, N. L. Welch, B. Stewart, A. Peretti, G. J. Starrett, Y.-Y. S. Pang, A.

Varsani, S. R. Krishnamurthy, P. A. Pesavento, D. H. McDermott, P. M. Murphy, J. L. Whited, B. Miller, J. M. Brenchley, S. P. Rosshart, B. Rehermann, J. Doorbar, B. A. Ta'ala, O.

600

601 Pletnikova, J. Troncoso, S. M. Resnick, A. M. Segall and C. B. Buck (2019). "Discovery of several thousand highly diverse circular DNA viruses." 555375.

Van Doorslaer, K., Z. Li, S. Xirasagar, P. Maes, D. Kaminsky, D. Liou, Q. Sun, R. Kaur, Y. Huyen and A. A. McBride (2017). "The Papillomavirus Episteme: a major update to the papillomavirus sequence database." Nucleic Acids Res 45(D1): D499-d506.

606 Vragniau, C., J. M. Hubner, P. Beidler, S. Gil, K. Saydaminova, Z. Z. Lu, R. Yumul, H. Wang, M. Richter, P. Sova, C. Drescher, P. Fender and A. Lieber (2017). "Studies on the Interaction of Tumor-Derived HD5 Alpha Defensins with Adenoviruses and Implications for Oncolytic Adenovirus Therapy." J Virol 91(6).

610 Wen, C. M., M. M. Chen, C. S. Wang, P. C. Liu and F. H. Nan (2015). "Isolation of a novel polyomavirus, related to Japanese eel endothelial cell-infecting virus, from marbled eels,

612 Yutin, N., S. Shevchenko, V. Kapitonov, M. Krupovic and E. V. Koonin (2015). "A novel group

613 of diverse Polinton-like viruses discovered by metagenome analysis." BMC Biol 13: 95.

614 Zallot, R., N. O. Oberg and J. A. Gerlt (2018). "'Democratized' genomic enzymology web tools for functional assignment." Curr Opin Chem Biol 47: 77-85.

616 Zimmermann, L., A. Stephens, S. Z. Nam, D. Rau, J. Kübler, M. Lozajic, F. Gabler, J. Söding, 617 A. N. Lupas and V. Alva (2017). "A Completely Reimplemented MPI Bioinformatics Toolkit 618 with a New HHpred Server at its Core." J Mol Biol S0022-2836(17): 30587-30589. 